\title{
Two components in IOR: evidence for response bias and perceptual processing delays using the SAT methodology
}

\author{
Yuanyuan Zhao • Dietmar Heinke • Jason Ivanoff • \\ Raymond M. Klein • Glyn W. Humphreys
}

Published online: 26 July 2011

(C) Psychonomic Society, Inc. 2011

\begin{abstract}
Inhibition of return (IOR) occurs when reaction times (RTs) are slowed to respond to a target that appears at a previously attended location. We used the speed-accuracy trade-off (SAT) procedure to obtain conjoint measures of IOR on sensitivity and processing speed by presenting targets at cued and uncued locations. The results showed that IOR is associated with both delays in processing speed and shifts in response criterion. When the target was briefly presented, the results supported a criterion shift account of IOR. However, when the target was presented until response, the evidence indicated that, in addition to a response bias effect, there was an increase in the minimal time required for information about the target to accumulate above chance level. A hybrid account of IOR is suggested that describes effects on both response bias and perceptual processing.
\end{abstract}

Keywords Inhibition of return - Math modelling ·

Decision making

The visual environment contains multiple stimuli that cannot be responded to at once. As a consequence, attentional mechanisms are required to select the behaviourally relevant stimuli and to filter out stimuli that are less

Y. Zhao $(\bowtie) \cdot$ D. Heinke $\cdot$ G. W. Humphreys

School of Psychology, University of Birmingham,

Birmingham, England, UK

e-mail: yuanyuan.a.zhao@gmail.com

J. Ivanoff

Department of Psychology, Saint Mary's University,

Halifax, Nova Scotia, Canada

R. M. Klein

Psychology Department, Dalhousie University,

Halifax, Nova Scotia, Canada relevant to our goals. One classical experimental approach used to study visual attention is to use spatial cueing to bias attention towards or away from a target (Posner \& Cohen, 1984). In this procedure, participants typically see a spatial cue either to the left or right side of fixation, followed by a target either at the same location as the cue or on the opposite side. Participants are asked to press a key as soon as they detect the target. When there are relatively short time intervals (SOAs) between the cue and the target, reaction times (RTs) are faster when the target and the cue are at the same location, as compared to when these stimuli appear on opposite sides. However, when the time interval between the cue and target is increased, this facilitatory effect of cueing is usually reversed: Participants are slower to respond when the target and cue appear at the same location than when they appear on opposite sides. Posner, Rafal, Choate, and Vaughan (1985) termed this the inhibition of return (IOR) effect.

One (standard) interpretation of these results is that the early facilitation effect reflects the fast, reflexive orienting of attention towards the sudden appearance of the cue, which leads to more efficient processing of targets at that location (Folk, Remington, \& Johnston, 1992; Posner, 1980; Posner \& Cohen, 1984; Yantis \& Hillstrom, 1994; Yantis \& Jonides, 1984). However, the nature of the later IOR effect remains unclear, with arguments having been made that IOR reflects either impaired perceptual processing (e.g., Cheal, Chastain, \& Lyon, 1998; Handy, Jha, \& Mangun, 1999; Lupiáñez, Milan, Tornay, Madrid, \& Tudela, 1997; Prime \& Ward, 2004) or a change in response criterion to stimuli at the cued location (e.g., Ivanoff \& Klein, 2001, 2003, 2004; Klein \& Taylor, 1994), or both (Ivanoff \& Klein, 2006). In this article, we aim to examine these two aspects of IOR using the speed-accuracy tradeoff (SAT) methodology. 
To assess IOR, we took a signal detection theory (SDT) approach. SDT separates processing of stimuli into two stages: a perceptual-coding stage and a decision stage (Green \& Swets, 1966; Swets, Green, Getty, \& Swets, 1978). Crucially, the participant's sensitivity to the stimulus can be measured with the $d^{\prime}$ metric, and the bias for responding in a particular direction may be determined with $c$, the decision criterion metric (Macmillan \& Creelman, 2005). Both measurements are influenced by several factors, including the quality of the stimuli and the length of time available for stimulus classification. Especially important for this article is the fact that the timing of the response to a stimulus reflects the monotonically increasing quality of perceptual encoding based on continued accumulation of information about the target, leading to the well-established SAT function (Reed, 1973; Wickelgren, 1977). Figure 1 illustrates three hypothetical SAT functions that might underlie the IOR effect. Within the framework of SDT, the IOR effect can be explained in at least two ways. One possibility is that IOR influences perceptual coding, with perceptual sensitivity to the target being impaired. For example, the maximal amount of information that can accrue from the time-limited presentation of the target may be less at regions affected by IOR (Fig. 1a). Alternatively, it may take longer to accumulate information about the target at the previously cued location (Fig. 1b). This is the attentional/perceptual account of IOR (cf. Handy et al., 1999; Ivanoff \& Klein, 2006; Reuter-Lorenz, Jha, \& Rosenquist, 1996). A second possibility is that IOR occurs because the criterion for the decision process is raised at the previously cued location - the criterion shift account (Ivanoff \& Klein, 2001, 2003, 2004, 2006; see Fig. 1c). Crucially, these two accounts predict different effects of IOR on performance accuracy, even if similar effects are predicted on RTs. The criterion shift account holds that there is no difference in the accumulation of information at the cued and uncued locations, but that cueing generally slows RT when a decision criterion is applied (it is applied later to targets at cued locations). Because the decision criterion is applied later, there should be more perceptual information available when a decision is made (at least when the perceptual information remains available for report). It follows that there should be higher accuracy at the cued than at the uncued location. In contrast, the attentional/perceptual account suggests that accuracy should be lower at the cued location, since information accrual is delayed or the rate of accrual is slowed there. Importantly, there is support for both accounts in the literature. We first will review the studies supporting the attentional/perceptual account.

Lupiáñez et al. (1997) investigated IOR in a discrimination task. They found that both delayed RTs and reduced accuracy were elicited at cued as compared to uncued locations across a range of SOAs from 700 to $1,300 \mathrm{~ms}$. Cheal et al. (1998) and Handy et al. (1999) also found decreased accuracy and delayed RTs when the cue shared the same location as the target. However, these studies used either masking or short target presentations, which might have led to a rapid loss of stimulus information. If response selection is delayed while stimulus information decays, performance may be less accurate under IOR conditions simply because of the greater decay of stimulus-based evidence over a longer period of time (see Ivanoff \& Klein, 2001, 2006, for a similar argument). This argument is supported by Handy et al.'s (1999) study, where $d^{\prime}$ was reported to decrease with longer RTs. In this study, for which participants were instructed to emphasise accuracy, they tended to respond more slowly and, interestingly, also with decreased accuracy.
A. Difference in asymptotic sensitivity

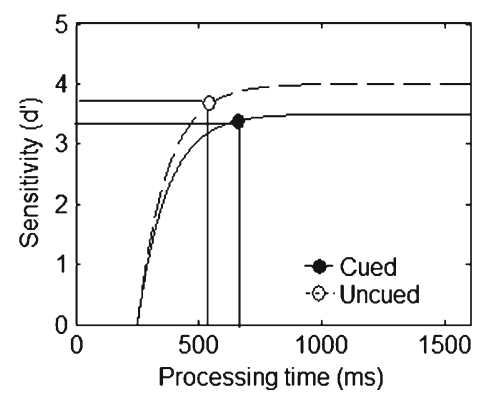

B. Difference in speed of information processing

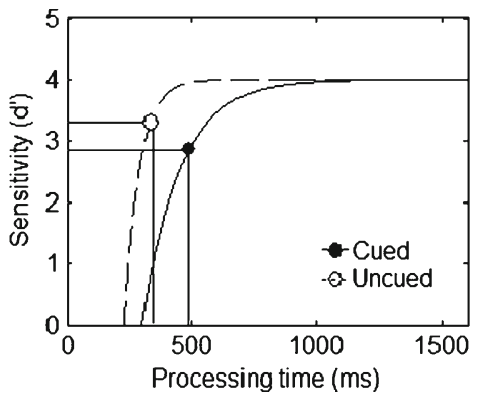

C. Criterion shift

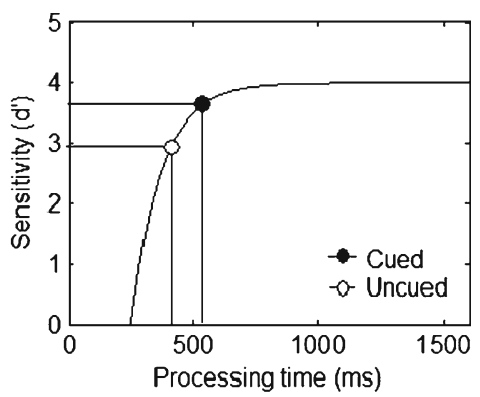

Fig. 1 Hypothetical SAT functions: $d^{\prime}$ sensitivity as a function of processing time. (a) Expected curves if IOR impairs only the asymptote sensitivity reached by a stimulus. The functions maintain the same intercept and rate of information processing, but differ in asymptote. (b) Expected curves if IOR affects the rate of information processing and the starting point at which stimulus information accumulates above chance. (c) Expected curves if IOR generates a criterion shift only. The figures show that the RT advantage for uncued over cued locations can be associated with changes in sensitivity (a), the speed or the starting point of information processing (b), or a criterion shift (c). Note that RTs and/or $d^{\prime}$ measures are determined by the balance of speed and accuracy that participants adopt 
Support for the criterion shift account of IOR comes from a series of studies by Ivanoff and colleagues (e.g., Ivanoff \& Klein, 2001, 2003, 2004, 2006; Klein \& Taylor, 1994). These studies reported a lower false alarm rate along with longer RTs for targets appearing at cued relative to uncued locations in both go/no-go and discrimination tasks. These authors argued that a lower false alarm rate implies a more conservative response criterion and therefore constitutes evidence for a criterion shift account of IOR. Although lower false alarms alone do not provide conclusive evidence for a criterion shift, as false alarms need to be considered together with the hit rate to properly measure criterion levels, $c$ scores in these studies were highly related to false alarms, as generally no misses were found. It is noteworthy that in these studies, targets were presented until response, thereby avoiding effects of stimulus decay on performance - unlike studies supporting the perceptual account.

The present study aimed to go beyond the above studies by fully measuring the SAT function so as to derive the parameter estimates for the intercept, slope, and asymptotes, as suggested by Wickelgren (1977). This approach enables the time course of information accrual to be plotted while, at the same time, allowing the experimenter to control the relations between the speed and accuracy of responses. Importantly, the procedure also allows us to determine the time course of target processing for limited and unlimited presentation times together, since this procedure produces false alarms together with hits at a reasonable rate for both. Importantly, in the case of limited target presentation time, the procedure allows us to determine whether effects of information decay contribute to performance. Finally, we also manipulated the luminance of the cues to test the generality and robustness of our findings with respect to cue luminance.

The SAT methodology has previously been applied in a variety of areas in cognitive psychology, including attentional cueing (Carrasco \& McElree, 2001; Giordano, McElree, \& Carrasco, 2009) and memory (Reed, 1973). The cited studies showed that the relationship between processing time and accuracy can best be described with the so-called SAT function. The SAT function assumes that response accuracy increases with processing time in an exponential fashion (see Fig. 1 for illustrations, and the Results section of Exp. 1 for the mathematical formula). The function has three parameters. The intercept parameter indexes the point in time at which the increase begins and accuracy departs from chance level. The sensitivity asymptote specifies the maximal possible accuracy. Finally, the rate parameter describes the growth rate of accuracy over time. The combination of the intercept and rate parameters can be interpreted as describing the speed of information processing (e.g., Giordano et al., 2009). Note that alternative models such as the linear ballistic accumulation (LBA) model proposed by Brown and Heathcote (2008) can also successfully accommodate decision making and choice response time (e.g., Farrell, Ludwig, Ellis, \& Gilchrist, 2010). In this model, evidence for alternative responses is independently accumulated in a linear manner, at different rates. Each accumulator begins with a starting amount of evidence, and a response is made when the first accumulator reaches the threshold. However, by fitting an SAT function here, we are able to compare our results to the results from similar studies using this method (e.g., Carrasco \& McElree, 2001; Ivanoff \& Klein, 2006).

Carrasco and McElree (2001) applied the SAT method to an investigation of the effect of spatial cueing on visual search. They found that cueing not only improved asymptotic sensitivity, but also accelerated the rate of information-processing speed at the cued location (at least at short cue-target SOAs). McCormick and Francis (2005) also found that spatial cueing improved processing speed at short SOAs. In contrast to these studies on early facilitatory cueing, Ivanoff and Klein (2006) explored IOR using of the SAT procedure. They examined sensitivity as a function of RT in both go/no-go and discrimination tasks using a time window procedure (Carrasco \& McElree, 2001; Ivanoff \& Klein, 2006; Wickelgren, 1977). Participants were required to respond within a 210 -ms time window that occurred 120 , 240,360 , or $480 \mathrm{~ms}$ after target presentation. In both tasks, IOR was observed in RTs across all but the longest time interval between the cue and the target $(480 \mathrm{~ms})$. This effect on RTs was accompanied by reduced $d^{\prime}$ at the cued locations for all but the shortest time interval $(120 \mathrm{~ms})$. No effect of a criterion shift was found except at the shortest time interval, when the criterion was higher for targets at cued relative to uncued locations. Together, these findings support the perceptual as well as the criterion shift accounts of IOR. The authors suggested that, early on in target processing, when the quality of stimulus evidence is poor, IOR is initially implemented as a criterion shift that biases participants against responding to targets at cued locations. However, later in time, when the quality of stimulus evidence improves, IOR appears to act upon perception, reducing sensitivity to cued targets and slowing RTs. The design of Ivanoff and Klein's (2006) study did not allow them to fit an SAT function, as they used only four intervals between target presentation and the response window. It is possible, however, that their effect on perceptual sensitivity could arise for at least two reasonsan effect on the asymptotic level of sensitivity at cued and uncued locations, or an effect on the time course of the buildup of perceptual information (see Fig. 1a and b for an illustration). The design of the present experiments overcomes this limitation by using seven time intervals ranging from 90 to $1,350 \mathrm{~ms}$, to enable SAT functions to be plotted and asymptote and rate effects to be distinguished. In 
addition, Ivanoff and Klein (2006) used only unlimited target presentation times, yet different effects might emerge with short and prolonged target exposures. Here we will apply the time window procedure to both unlimited and limited target presentations to explore whether differences in target exposure contribute to the conflicting results found in the IOR literature. We used limited target presentation in the first experiment and fitted a single SAT function for the cued and uncued conditions (Fig. 1c). In Experiment 2, unlimited target presentations were used, and there was a delay in information processing for the cued relative to the uncued target (Fig. 1b). In addition, both experiments showed a more conservative criterion at the cued than at the uncued location.

\section{Experiment 1: brief target presentation}

In Experiment 1, a brief target presentation was used. With brief target presentations, Lupiáñez et al. (1997), Cheal et al. (1998), and Handy et al. (1999) all found evidence indicating an effect of IOR on perception, since accuracy increased at cued relative to uncued locations. However, their studies did not control for speed-accuracy trade-offs, nor did they determine the SAT function to assess the precise effect of IOR on performance. Our experimental procedure follows Ivanoff and Klein's (2006) second experiment, the main differences being that Ivanoff and Klein (2006) had used an unlimited target presentation and four response windows. Here, we limited the presentation of the target and used seven response windows so that we were able to fit a complete SAT function. We also manipulated target luminance. Hunt and Kingstone (2003) and Reuter-Lorenz et al. (1996) reported that reductions in target luminance increased IOR. Target Luminance was manipulated as a between-subjects factor to test the generality of our findings and to assess whether there were differential effects of this factor across the SAT function.

\section{Method}

Participants A group of 17 postgraduates participated (8 were tested with the bright target and 9 with the dim target), comprising 10 females and 7 males from 22 to 40 years of age, with a mean age of 28.5 years. The participants were from the psychology department of Dalhousie University, and they were naive as to the purpose of the study. The participants were paid Can\$25 and took part in approximately two sessions of $70 \mathrm{~min}$ each, which were completed on different days within the same week. All participants reported normal or corrected-to-normal vision, and all except 3 were right-handed.
Apparatus Stimulus presentation and data collection were performed using E-Prime 1.2. The stimuli were presented on a 17-in Viewsonic PT775 monitor controlled by a personal computer, and responses were polled from the standard keyboard.

Stimuli The stimulus display (see Fig. 2) consisted of a fixation circle $\left(68.86 \mathrm{~cd} / \mathrm{m}^{2}\right)$ subtending $0.6^{\circ}$ in diameter and $0.07^{\circ}$ thick, presented at the centre of the screen (background $0.82 \mathrm{~cd} / \mathrm{m}^{2}$ ), and two outline boxes with asterisks $\left(2.5 \mathrm{~cd} / \mathrm{m}^{2}\right)$ in the centres of the boxes, aligned horizontally to the left and right of the fixation cross. The distance between the fixation circle and the centre of each box was $7.1^{\circ}$. Each box had a $0.15^{\circ}$-thick frame and subtended $3.3^{\circ}$ in height and $3.0^{\circ}$ in width. Each asterisk was made from overlapping " $\times$ " and " + " signs; the "+" was $2.3^{\circ}$ in length and $0.2^{\circ}$ thick, and the " $\times$ " was $3.4^{\circ}$ in length and $0.15^{\circ}$ thick, with both elements containing the same number of pixels. The cue comprised a luminance increase of one of the box outlines to $123.69 \mathrm{~cd} / \mathrm{m}^{2}$ (for the bright cue) or $23.30 \mathrm{~cd} / \mathrm{m}^{2}$ (for the dim cue), each lasting $80 \mathrm{~ms}$. The cue back to fixation comprised a thickening of the fixation circle to $0.15^{\circ}$ for $100 \mathrm{~ms}$. The target was a luminance increase of either the "+" or the " $x$ " to $123.69 \mathrm{~cd} / \mathrm{m}^{2}$ (the bright target) or to $23.30 \mathrm{~cd} / \mathrm{m}^{2}$ (the dim target) for $80 \mathrm{~ms}$.

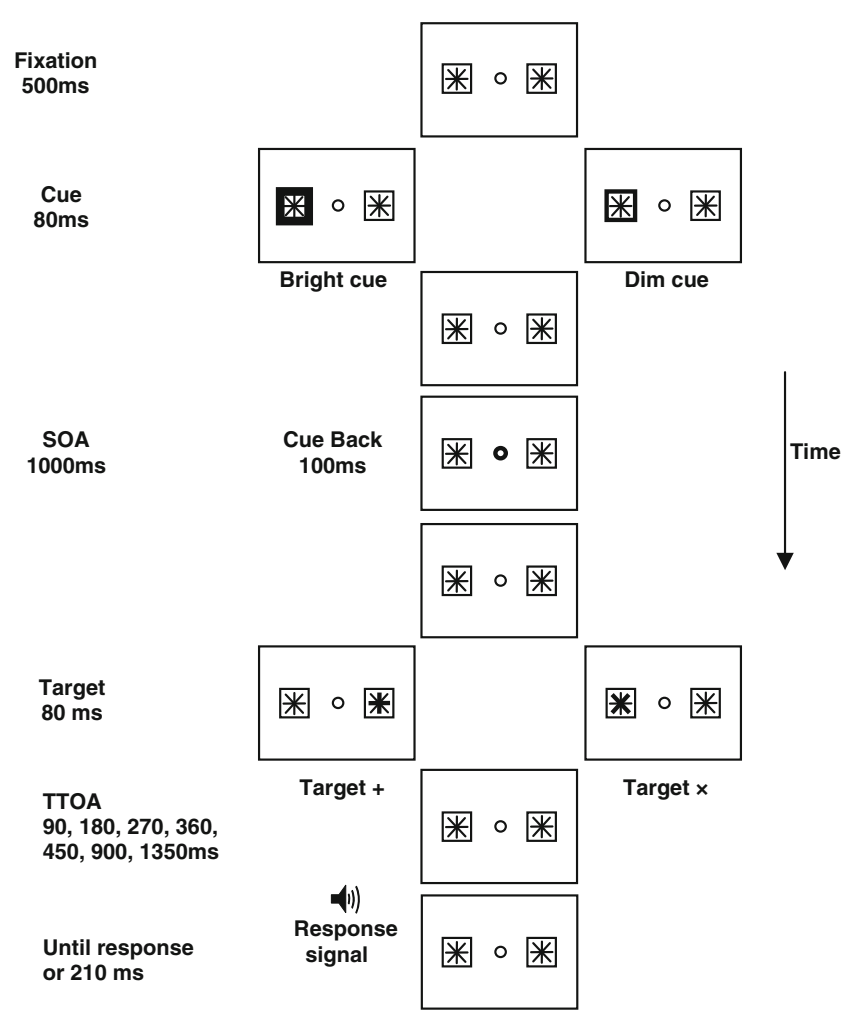

Fig. 2 The trial sequence used in Experiment 1 
Procedure The experiment was conducted in a quiet, dimly lit room. Participants were given both written and oral instructions for the task, and were individually tested sitting at a distance of approximately $57 \mathrm{~cm}$ from the computer screen. RTs and response accuracy were recorded by the computer. The participants were instructed to maintain fixation throughout the experiment and not to make any eye movements. They were also told that cues were uninformative as to the location of the target.

The trial sequence is shown in Fig. 2. Each trial began with a blank 500-ms intertrial interval, not shown in Fig. 2. Following this, a display consisting of a central fixation circle and two peripheral boxes with asterisks in the middle was presented for $500 \mathrm{~ms}$. Subsequently, one of the peripheral boxes was cued by increasing the luminance of the outline of the box for $80 \mathrm{~ms}$. This increase was experienced as a flash. The cue comprised two levels of luminance changes. After the cue was removed, the fixation circle and the two boxes with asterisks were presented alone for $170 \mathrm{~ms}$. A return cue was presented by enlarging the fixation circle for $100 \mathrm{~ms}$ to ensure that attention did not remain at the cued location. The fixation circle and two boxes with asterisks were then presented alone for $650 \mathrm{~ms}$. Finally, the target was presented as a luminance increase of the "+" or the " $\times$ " in the left or the right box. Target presentation time was $80 \mathrm{~ms}$ and comprised two levels of luminance change. The targets, the "+" or " $\times$," appeared with equal frequency.

On each day, each of the seven target-tone onset asynchronies (TTOAs; 90, 180, 270, 360, 450, 900, or $1,350 \mathrm{~ms}$ ) was presented in a separate block. Whether TTOAs are blocked or mixed appears to have little effect on the SAT function (Miller, Sproesser, \& Ulrich, 2008). The order of the TTOAs was randomised. The tone (a high pitch) signalled a 210-ms response window in which participants were instructed to make the appropriate response. Half of the participants were instructed to make a keypress with the index finger of their left hand on the "Z" key and the middle finger on the " $A$ " key, and the other half were instructed to use their right index finger on the "M" key and middle finger on the "K" key. Within each group, half were also instructed to press the index finger whenever "+" was presented and to press the middle finger whenever " $\times$ " was presented, or vice versa for the other half. Detection time for the target was measured from the target onset to response. If no response was made within the response window, an error tone (low pitch) was given at the end of each response window to inform participants; this provided no information about whether the responses were correct.

After the instructions had been understood, the participants were administered practice trials until they were able to perform the task correctly. The responses for these trials were not recorded.
Design The experiment consisted of a 2 (cue luminance: bright/dim) $\times 2$ (cue: cued/uncued) $\times 7$ (TTOA: 90/180/ 270/360/450/900/1,350 ms) $\times 2$ (target luminance: bright/ dim) mixed design with Target as a between-subjects factor. The experiment consisted of 1,792 trials in total, which were divided into 14 blocks, and the first 16 trials of each block were excluded from analyses. Within each block, the trials were randomised with respect to trial type and were equally divided with respect to cue luminance, cue, SOA, and target location. Each of the experimental conditions contained 56 trials, with 28 trials using a target " + " and 28 trials with a target " $\times$ ".

Data analysis Sensitivity was calculated as

$d^{\prime}=z(H)-z(F)$

where $z(H)$ is the $z$ score for correct responses to "+" (hits) and $z(F)$ is the $z$ score for incorrect responses to " $\times$ " (false alarm). Extreme values of hits and false alarms were treated by subtracting or adding .5 to the counts, respectively (loglinear transformation; Snodgrass \& Corwin, 1988).

The criterion was given by

$c=-[z(H)+z(F)] / 2$

The criterion value in a discrimination task only indicates bias towards a particular response, either "+" or " $\times$." To determine additional evidence for a criterion shift, we used the following indirect measures: anticipations, response frequencies, and misses (see Ivanoff \& Klein, 2006). Anticipations are the percentages of responses after target onsets but before the response signal. Response frequency refers to the percentage of responses within the response window (including correct and wrong responses). Misses are either responses made after the response window or failures to respond on a trial (time limit: $500 \mathrm{~ms}$ after the response signal). A more conservative criterion is reflected by an increase of misses and a decrease of anticipations. The IOR effect on RTs was measured based on a tone reaction time (tone RT) analysis. The tone RT is the time from the response signal (tone) to the response when a correct keypress occurred within the response window.

The dependent variables were analysed using ANOVAs, with the Greenhouse-Geisser correction applied when Mauchly's test of sphericity was significant.

\section{Results}

The hit rate (response rate within the response window) was $79.05 \%$ on average, with a minimum of $68.62 \%$. The accuracy rate of hits was $84.75 \%$ on average with a minimum of $75.87 \%$. Figure 3 shows that $d^{\prime}$ increased 
with increasing processing time. Hence, despite our use of a brief target presentation, information about the target increased rather than decayed as the TTOA was increased. Since the time course of $d^{\prime}$ is very similar to that from other experiments employing the SAT methodology (e.g., Carrasco $\&$ McElree, 2001), we fitted the SAT function as suggested by Wickelgren (1977).

Fit of SAT function We used the following mathematical description of the SAT function to fit the data of $d^{\prime}$ for each TTOA condition and average processing time (TTOA plus RTs for correct responses) for that condition (Liu \& Smith, 2009; Wickelgren, 1977):

$d^{\prime}(t)=\left\{\begin{aligned} & \lambda\left(1-e^{-(t-\delta) / \beta}\right), t>\delta \\ & 0 \quad, t \leq \delta\end{aligned}\right.$

where $\lambda$ is the asymptotic parameter reflecting the accuracy with maximal processing time, $1 / \beta$ describes the rate at which accuracy grows from chance $\left(d^{\prime}=0\right)$ to asymptote, and $\delta$ is an intercept parameter indexing the discrete point in time when accuracy departs from chance.

We used a hierarchical model-testing scheme to find the most parsimonious SAT model for each participant (Carrasco \& McElree, 2001; Giordano et al., 2009; Liu \& Smith, 2009). The model selection procedure began with the full model, where each condition was modelled with a unique set of parameters. To find the simplest model, we used the $G^{2}$ statistic (the difference in deviance values between two nested models; Liu \& Smith, 2009). The maximum likelihood criterion was used to fit the models with MATLAB'S fminsearch (Liu \& Smith, 2009). In addition, the resulting model was characterised with adjusted $R^{2}$ (Dosher, Han, \& Lu, 2004):

$\operatorname{adj} R^{2}=1-\frac{\sum_{i=1}^{n}\left(d_{i}-\widehat{d}_{i}\right)^{2} /(n-k)}{\sum_{i=1}^{n}\left(d_{i}-\bar{d}\right)^{2} /(n-1)}$

where $d_{i}$ is the observed $d^{\prime}$ values, $\widehat{d}_{i}$ is the predicted $d^{\prime}$ values, $\bar{d}$ is the mean of $d^{\prime}, n$ is the number of data points, and $k$ is the number of free parameters.

To analyse the results across all participants, the parameters for each participant were entered into three separate three-way within-participants ANOVAs (Liu \& Smith, 2009).

The final models produced an average adjusted $R^{2}$ value of .930 across participants, with a minimum of .858 . The parameters of asymptote, intercept, and slope in the final model for each participant were entered into three separate $2 \times 2 \times 2$ ANOVAs with Cue Luminance (bright or dim) and Cue (cued or uncued) as within-subjects factors and Target Luminance (bright or dim) as a between-subjects factor. Figure 4 shows the means of the three sets of parameters. ${ }^{1}$ There were no significant effects of any factor for any of the parameters [asymptote: cue, $F(1,15)=0.20$, $p=.66$; intercept: cue, $F(1,15)=1.04, p=.32 ; 1 /$ slope: cue, $F(1,15)<0.001, p=.99$ ]. Figure 3 illustrates the means of the curve-fitting procedure for $d^{\prime}$ for each participant as a function of processing time, cue luminance, and cue. The mean data points for each condition are also shown. The pattern of the curve in Fig. 3 is consistent with pattern $\mathrm{C}$ in Fig. 1.

Tone RT The mean tone RTs for each condition are shown at the top of Table 1 . The tone RTs were entered into a $2 \times 2$ $\times 7 \times 2$ ANOVA with Cue Luminance (bright or dim), Cue (cued or uncued), and TTOA (90, 180, 270, 360, 450, 900, or $1,350 \mathrm{~ms}$ ) as within-subjects factors and Target Luminance (bright or dim) as a between-subjects factor. The main effect of cue was significant, $F(1,15)=52.69, p<$ .001: RTs were $4.74 \mathrm{~ms}$ faster in the uncued than in the cued condition. There was a Cue $\times$ TTOA interaction $[F(6,90)=$ $5.48, p<.001]$, reflecting greater IOR at early TTOAs. The main effect of TTOA was significant $[F(6,90)=25.19$, $p<.001$ ], indicating decreasing then increasing (a Ushaped function) RTs across the TTOAs. There was also a significant Cue Luminance $\times$ Target Luminance interaction $[F(1,15)=4.77, p<.05]$ (Fig. 5). When the target was $\mathrm{dim}, \mathrm{RTs}$ were slowed following the bright cue (131.28 ms) relative to the dim cue $(129.10 \mathrm{~ms})$; however, the data went in the opposite direction when the target was bright (bright cue, $125.85 \mathrm{~ms}$; dim cue, $126.51 \mathrm{~ms}$ ). None of the other main effects or interactions reached significance (all $p \mathrm{~s}>.1$ ).

We conducted separate ANOVAs with Cue Luminance and Cue as factors at each TTOA. ${ }^{2}$ Figure 6 shows the mean IOR (positive values indicate IOR) for each combination of cue luminance and TTOA (the between-subjects factor of Target Luminance was merged for all of the figures). There were significant IOR effects at TTOAs of $90 \mathrm{~ms}$ [magnitude $8.21 \mathrm{~ms} ; F(1,16)=19.45, p<.001], 180 \mathrm{~ms}$ [magnitude $13.67 \mathrm{~ms} ; F(1,16)=31.22, p<.001]$, and $270 \mathrm{~ms}$ [magnitude $4.61 \mathrm{~ms} ; F(1,16)=7.07, p<.05]$.

\footnotetext{
${ }^{1}$ The between-subjects factor Target Luminance was merged in the figure (the same holds for the other figures and tables in this experiment), as there was no major effect of target luminance. This makes the figures here consistent with the figures and tables in Experiment 2, where target luminance was not manipulated.

${ }^{2}$ To further explore the significant Cue $\times$ TTOA interaction, separate ANOVAs were performed at each TTOA. Moreover, in earlier research, Ivanoff and Klein (2006) showed that the tone RT, $d^{\prime}$, anticipation, response frequency, and miss measures behave differently at contrasting TTOAs, which they used as evidence of early criterion shifts and late perceptual effects of IOR as a function of the TTOA. Therefore, we performed separate ANOVAs at each TTOA for all these measurements for all experiments (even though some of the interactions were not significant) in order to examine whether these findings were replicated in the present study.
} 


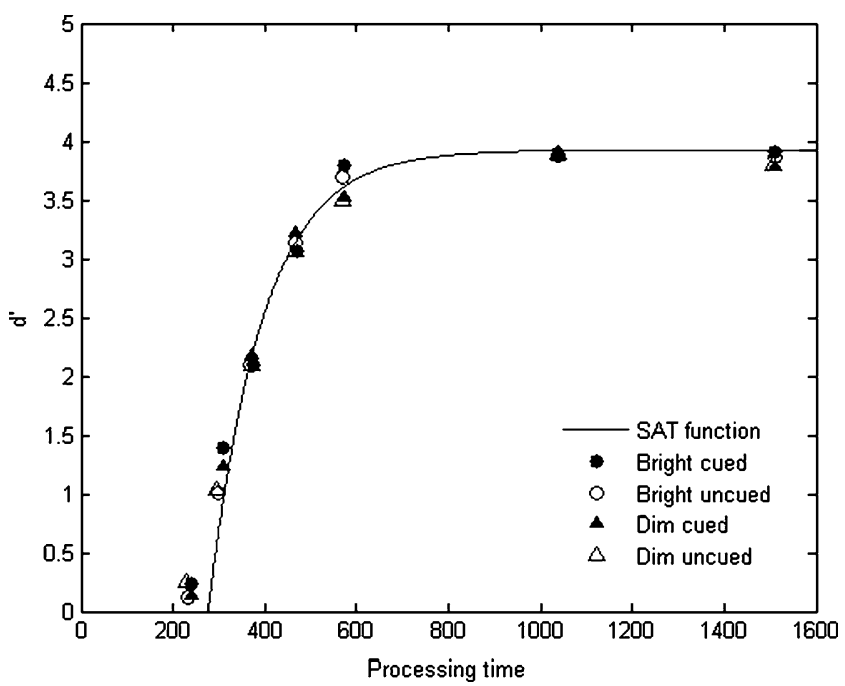

Fig. 3 Average sensitivity as a function of processing time for four conditions. A smooth function shows the average of the best-fitting model based on the three parameters of Eq. 1. The marked symbols show the mean data points for each condition across participants. Note that the curve was based on the average of parameters derived from fits for each individual but not for the mean data

Sensitivity (d') Table 1 shows $d^{\prime}$ for each condition. From the fitted SAT curve, it is clear that $d^{\prime}$ reached ceiling before TTOA $900 \mathrm{~ms}$. We therefore omitted TTOAs of 900 and $1,350 \mathrm{~ms}$ from the analyses. The same analysis was conducted in Experiment 2 below. The main effect of cue was significant $[F(1,15)=7.38, p<.05]$ : $d^{\prime}$ was 0.09 higher for targets at the cued than at the uncued location. There was also a significant TTOA main effect $[F(4,60)=$ $150.48, p<.001] ; d^{\prime}$ increased as TTOA increased from 90 to $450 \mathrm{~ms}$. None of the other main effects were reliable, and there were no interactions (all $p \mathrm{~s}>.1$ ).

Figure 7 shows the mean IOR in $d^{\prime}$ for each combination of cue luminance and SOA (a positive value indicates a lower $d^{\prime}$ at the cued location). Separate ANOVAs for Cue Luminance $\times$ Cue were performed at each TTOA. There was a significant main effect of cue at TTOA $180 \mathrm{~ms}[F(1$, $16)=6.67, p<.05]$, in that $d^{\prime}$ was 0.30 higher for cued trials than uncued trials. There was also a significant main effect of cue luminance at TTOA $450 \mathrm{~ms}[F(1,16)=7.61$, $p<.05]$; $d^{\prime}$ was 0.24 higher for the bright cue than for the dim cue.

Fig. 4 Mean parameters in Experiment 1: (Left) asymptote, (Middle) intercept, (Right) 1/slope
Anticipations Anticipations (responses after target onsets but before the response signal) for each condition, presented as percentages, are shown in Table 1. The main effect of cue was significant $[F(1,15)=5.01, p<.05]$; anticipations were $0.78 \%$ lower for targets at the cued than at the uncued location. The main effect of TTOA was significant $[F(2.92$, 43.86) $=15.34, p<.001]$; anticipations increased as the TTOA lengthened and were constant after a TTOA of 360 ms. Finally the Cue Luminance $\times$ Cue $\times$ Target Luminance interaction was also significant $[F(1,15)=$ $5.00, p<.05]$. Anticipations were lower for targets at the cued than at the uncued location when the cue and target luminances were both dim, but not with the other luminance combinations. None of the other main effects were reliable, and there were no interactions (all $p \mathrm{~s}>.08$ ).

Separate ANOVAs with the factors Cue Luminance and Cue at each TTOA showed a significant cue main effect at TTOA $270 \mathrm{~ms}[F(1,16)=16.29, p<.001]$ : There were $2.84 \%$ fewer anticipations made to the cued than to the uncued location, in this case.

Response frequency Table 1 shows the response frequencies (both correct and incorrect responses within the response window) as percentages in each condition. The main effect of TTOA was significant $[F(3.08,46.16)=6.65, p<.001]$. The response frequency was higher at TTOA $270 \mathrm{~ms}$ than at the other TTOAs. The Cue $\times$ TTOA interaction was also significant $[F(6,90)=4.94, p<.001]$. No other main effects or interactions were significant (all $p \mathrm{~s}>.1$ ).

Separate ANOVAs with the factors Cue Luminance and Cue were conducted for each TTOA. There was a significant main effect of cue at TTOA $90 \mathrm{~ms}[F(1,16)=$ $11.48, p<.01] ; 6.83 \%$ fewer responses were made to targets at the cued than at the uncued location. There were also main effects of cue at TTOA $270 \mathrm{~ms}[F(1,16)=4.64$, $p<.05]$; there were $2.42 \%$ more responses to targets at the cued than at the uncued location.

Misses The percentages of misses (responses after the response window or failures to respond on a trial) are also provided in Table 1. The main effect of cue was significant $[F(1,15)=13.04, p<.01]$, with $1.68 \%$ more misses to targets at cued than at uncued locations. The main effect of

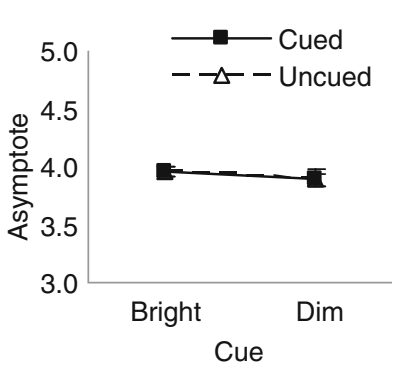

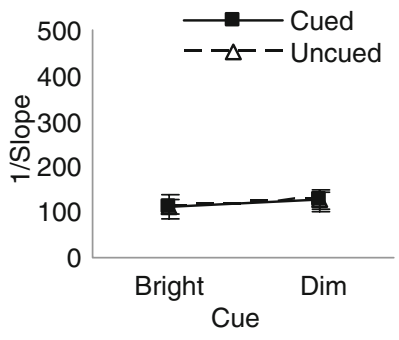


Table 1 Mean tone RTs, $d^{\prime}$, percentages of anticipations, percentages of response frequencies, percentages of misses, and criterion for each condition in Experiment 1

\begin{tabular}{|c|c|c|c|c|c|c|c|c|c|}
\hline & \multirow[t]{2}{*}{ Conditions } & & \multicolumn{7}{|c|}{ TTOA (ms) } \\
\hline & & & 90 & 180 & 270 & 360 & 450 & 900 & 1,350 \\
\hline \multirow[t]{4}{*}{ Tone RTs (ms) for correct responses } & \multirow[t]{2}{*}{ Bright cue } & Cued & 150.5 & 131.1 & 105.2 & 110.6 & 122.9 & 139.7 & 159.2 \\
\hline & & Uncued & 142.3 & 117.5 & 98.7 & 107.7 & 119.6 & 138.6 & 158.6 \\
\hline & \multirow[t]{2}{*}{ Dim cue } & Cued & 148.5 & 128.8 & 102.8 & 107.9 & 124.1 & 138.9 & 159.3 \\
\hline & & Uncued & 140.3 & 115.1 & 100.1 & 106.5 & 120.0 & 139.8 & 158.2 \\
\hline \multirow[t]{4}{*}{$d^{\prime}$} & \multirow[t]{2}{*}{ Bright cue } & Cued & 0.24 & 1.40 & 2.10 & 3.07 & 3.79 & 3.89 & 3.91 \\
\hline & & Uncued & 0.12 & 1.01 & 2.10 & 3.13 & 3.70 & 3.88 & 3.87 \\
\hline & \multirow[t]{2}{*}{ Dim cue } & Cued & 0.14 & 1.24 & 2.18 & 3.22 & 3.52 & 3.86 & 3.78 \\
\hline & & Uncued & 0.25 & 1.04 & 2.10 & 3.06 & 3.49 & 3.90 & 3.79 \\
\hline \multirow[t]{4}{*}{ Anticipations (\%) } & \multirow[t]{2}{*}{ Bright cue } & Cued & 1.68 & 0.95 & 1.47 & 10.92 & 9.77 & 10.19 & 7.46 \\
\hline & & Uncued & 1.58 & 1.05 & 4.31 & 11.45 & 10.92 & 10.71 & 7.88 \\
\hline & \multirow[t]{2}{*}{ Dim cue } & Cued & 1.89 & 0.53 & 2.63 & 10.40 & 10.50 & 10.19 & 9.03 \\
\hline & & Uncued & 2.52 & 1.26 & 5.46 & 12.39 & 11.03 & 8.51 & 9.66 \\
\hline \multirow[t]{4}{*}{ Response frequencies (\%) } & \multirow[t]{2}{*}{ Bright cue } & Cued & 72.58 & 81.30 & 89.18 & 78.36 & 76.16 & 79.83 & 75.21 \\
\hline & & Uncued & 79.31 & 83.93 & 86.76 & 77.73 & 77.21 & 78.47 & 74.47 \\
\hline & \multirow[t]{2}{*}{ Dim cue } & Cued & 70.06 & 81.72 & 87.92 & 77.73 & 78.89 & 78.68 & 74.16 \\
\hline & & Uncued & 77.00 & 83.72 & 85.50 & 77.31 & 74.89 & 81.41 & 73.84 \\
\hline \multirow[t]{4}{*}{ Misses (\%) } & \multirow[t]{2}{*}{ Bright cue } & Cued & 22.58 & 15.55 & 7.35 & 9.56 & 13.66 & 9.77 & 17.02 \\
\hline & & Uncued & 17.23 & 11.97 & 6.72 & 9.87 & 11.45 & 10.29 & 17.23 \\
\hline & \multirow[t]{2}{*}{ Dim cue } & Cued & 25.21 & 15.97 & 8.30 & 10.82 & 9.98 & 11.03 & 16.28 \\
\hline & & Uncued & 17.33 & 12.39 & 7.35 & 8.72 & 13.13 & 9.77 & 16.07 \\
\hline \multirow[t]{4}{*}{ Criterion } & \multirow[t]{2}{*}{ Bright cue } & Cued & .12 & .16 & -.01 & .02 & -.05 & .02 & .01 \\
\hline & & Uncued & .05 & .03 & .06 & .07 & .01 & -.05 & -.04 \\
\hline & \multirow[t]{2}{*}{ Dim cue } & Cued & .15 & .11 & .02 & .09 & -.02 & -.03 & -.04 \\
\hline & & Uncued & .02 & .13 & .08 & .10 & .07 & -.05 & .03 \\
\hline
\end{tabular}

The columns in bold font indicate that an ANOVA for the respective TTOAs found significant effects (see the text for details). Note that the criterion value in a discrimination task only indicates a bias towards a particular response. Here, a positive criterion value indicates a response bias towards "+," and negative value indicates a bias towards " $\times$ ".

TTOA was significant $[F(3.01,45.16)=11.60, p<.001]$, with misses decreasing and then increasing as TTOA increased. In addition, the Cue $\times$ TTOA interaction was significant $[F(3.57,53.56)=4.92, p<.01]$, as was the

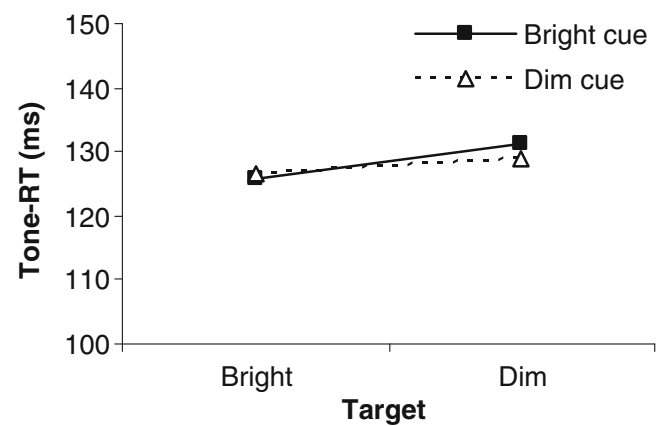

Fig. 5 The significant Cue Luminance $\times$ Target Luminance interaction of tone RT
Cue $\times$ TTOA $\times$ Target Luminance interaction $[F(6,90)=$ $2.96, p<.05]$. None of the other main effects or interactions reached significance (all $p \mathrm{~s}>.1$ ).

Separate ANOVAs with the factors Cue Luminance and Cue at each TTOA revealed the following: There were more misses at TTOA $90 \mathrm{~ms}[F(1,16)=12.07$, $p<.01]$ and TTOA $180 \mathrm{~ms}[F(1,16)=6.97, p<.05]$ to targets at cued as compared with uncued locations [effect sizes $6.62 \%$ and $3.57 \%$, respectively]. There was also a Cue Luminance $\times$ Cue interaction at TTOA $450 \mathrm{~ms}[F(1$, $16)=5.72, p<.05]$. More misses were made to targets at cued than at uncued locations when the cues were bright; however, this effect was reversed when the cues were dim.

Criterion (c) Finally, Table 1 shows the criterion measure for each condition. Criterion values reflect bias towards a particular response. The main effect of TTOA was significant 
Fig. 6 IOR on RTs in Experiment 1 . Positive values indicate IOR on RTs

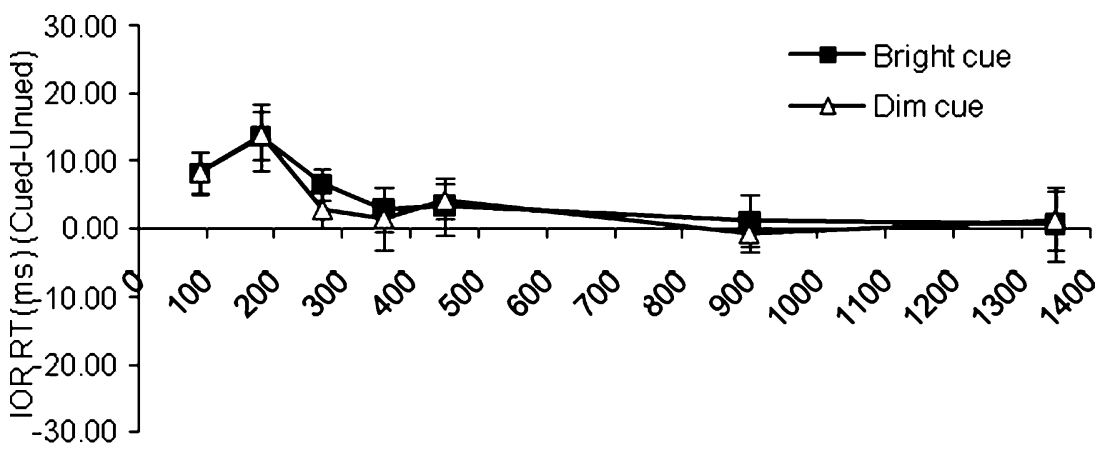

Processing time (ms)
$[F(3.75,56.28)=3.03, p<.05]$, with $c$ decreasing (from positive to negative values) as TTOA increased. There was a slightly greater bias to " + " rather than " $\times$ " at early TTOAs, but this was reversed at late TTOAs. None of the other main effects or interactions reached significance (all $p s>.1$ ).

\section{Discussion}

The results showed that the quality of target information as measured by $d^{\prime}$ increased with increasing processing time (i.e., at longer TTOAs). This accrual of target information took place despite the brief target presentation, providing no evidence that target decay was a critical factor in this study. This time course for $d^{\prime}$ is similar to those from previous experimental findings with the SAT methodology (e.g., Carrasco \& McElree, 2001). Nevertheless, our curve-fitting procedure did not reveal significantly different parameters across the different experimental conditions; instead, all conditions followed the same SAT function.

There was evidence for IOR in terms of RTs, especially for short TTOA intervals (TTOAs of 90, 180, and $270 \mathrm{~ms}$ ). These results are largely consistent with findings by Ivanoff and Klein (2006), who also demonstrated an IOR effect for early response windows. The results also showed a significant overall effect of cueing on $d^{\prime}$, with greater sensitivity for targets at cued than at uncued locations. In the absence of any difference in the parameters of the SAT function, this pattern is consistent with the criterion shift account of IOR (see Fig. 1c). Also note that the results were largely robust to changes in cue and target luminance.

Converging evidence for a criterion shift was also found from the analysis of anticipations, response frequencies, and misses. Participants tended to make fewer anticipations and more misses to targets at the cued location than at uncued locations, particularly at short TTOAs. There were no cueing effects on the response frequency measures. This pattern of responses indicates that participants were more conservative when responding to targets at cued relative to uncued locations. Finally, the manipulation of cue and target luminance had little impact on the results.

The present evidence for a criterion shift account of IOR contradicts at least one conclusion of Ivanoff and Klein (2006), who found that $d^{\prime}$ was significantly decreased at the cued as compared with the uncued location-a result consistent with a perceptual account of IOR. However, there is at least one major methodological difference between our study and that of Ivanoff and Klein (2006): Our target appeared for $80 \mathrm{~ms}$, while theirs was presented until the response. In the next experiment, we will test whether this difference was responsible for the contrasting findings. ${ }^{3}$

\section{Experiment 2: unlimited target presentation times}

The second experiment investigated whether the target presentation duration was critical to the differences between our data and those of Ivanoff and Klein's (2006). In this case, the target presentation time was unlimited, making our experiment essentially a replication of Ivanoff and Klein (2006), only with more TTOAs.

\section{Method}

The method was the same as for the first experiment, except where mentioned below.

Participants A group of 10 volunteers, 9 females and 1 male, from 18 to 28 years of age were recruited. The participants were naive as to the purpose of the study, and none had taken part in the previous experiment. All participants reported normal or correct-to-normal vision, and all were right-handed.

\footnotetext{
${ }^{3}$ There was also another methodological difference as compared to Ivanoff and Klein (2006): They used a target onset instead of a luminance change, which may have been responsible for our partial failure to replicate their results. We tested this idea in a separate experiment, but did not find a reliable difference between that experiment and Experiment 1.
} 
Fig. 7 IOR on $d^{\prime}$ in Experiment 1. Positive values indicated lower $d^{\prime}$ at the cued location

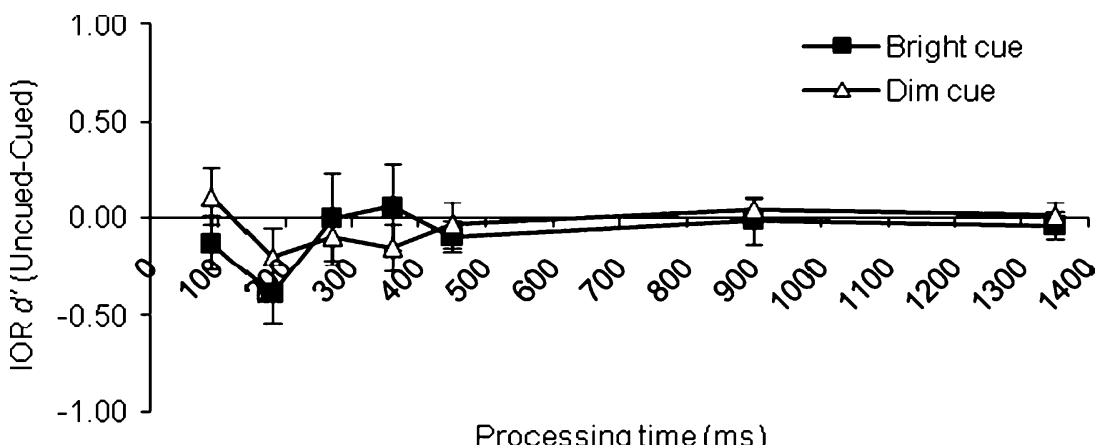

Stimuli Since the effects in the previous experiment were immune to the difference in the two levels of cue luminance, we decided to increase the cue thickness to $0.4^{\circ}{ }^{4}$ The luminance values remained the same as in Experiment 1, except that the target luminance was always dim, as Experiment 1 did not show an effect of target luminance. The target remained visible until a response was made or the response window had elapsed.

Results

The mean hit rate was $76.06 \%$, with a minimum of $67.73 \%$. The accuracy rate of hits was $84.52 \%$ per participant on average, with a minimum of $78.10 \%$. The SAT function was fitted and the data were analysed as before.

SAT function The average adjusted $R^{2}$ across participants was .894 , with a minimum of .767 . Figure 8 shows the means of the three sets of parameters. An ANOVA revealed a significant main effect of cue on the intercept parameter $[F(1,9)=6.00, p=.037]$; the time at which information accumulation rose above chance was delayed $18.17 \mathrm{~ms}$ for targets at the cued as compared with the uncued location. None of the other effects or interactions were significant [asymptote: cue luminance, $F(1,9)=$ $0.00, p=.99$; cue, $F(1,9)=0.50, p=.50$; interaction, $F(1,9)=1.02, p=.34$; intercept : cue luminance, $F(1,9)=$ $1.86, p=.21$; interaction, $F(1,9)=1.86, p=.21 ; 1 /$ slope: cue luminance, $F(1,9)=2.15, p=.18$; cue, $F(1,9)=0.40$, $p=.54$; interaction, $F(1,9)=0.48, p=.51]$. The mean curve fits and data points for each condition are presented in Fig. 9. The pattern of the curves in Fig. 9 is consistent with pattern B in Fig. 1.

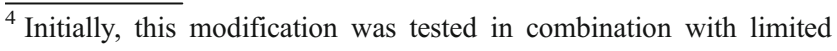
onset target presentation to further examine the generality and robustness of our findings. However, we did not find an effect in that separate experiment. For consistency reasons, we maintained the larger cue size in the present experiment.
}

Tone $R T$ The main effect of cue was significant $[F(1,9)=$ $7.59, p<.05]$ (Table 2). RTs were $5.67 \mathrm{~ms}$ slower for targets at cued than at uncued locations. The main effect of TTOA was significant $[F(6,54)=33.41, p<.001]$; there was a $\mathrm{U}$ shaped function relating overall RT to TTOA. In addition, the Cue $\times$ TTOA interaction was significant $[F(6,54)=$ $10.39, p<.001]$. IOR appeared at the earlier TTOAs and disappeared at later TTOAs (see Table 2). None of the other main effects or interactions reached significance [Cue Luminance $\times$ Cue, $F(1,9)=0.48, p=.50$; Cue Luminance $\times$ Cue $\times$ TTOA, $F(6,54)=0.15, p=.99]$.

Figure 10 shows the mean IOR for all combinations of cue luminance and SOA. Separate ANOVAs with the factors of Cue Luminance and Cue were performed at each TTOA. There were significant effects of cue at TTOAs of $90 \mathrm{~ms}[F(1,9)=14.80, p<.01]$ and $180 \mathrm{~ms}[F(1,9)=$ 23.97, $p<.01]$ (magnitude of IOR effects: 13.82 and $20.48 \mathrm{~ms}$, respectively). There was a main effect of cue luminance at TTOA $450 \mathrm{~ms}[F(1,9)=7.58, p<.05]$, with RTs slower after a bright than after a dim cue (a $5.15-\mathrm{ms}$ effect).

Sensitivity $\left(d^{\prime}\right)$ The data from TTOAs of 90 to $450 \mathrm{~ms}$ were entered into an ANOVA (Table 2). The main effect of TTOA was significant $[F(4,36)=141.65, p<.001]$; $d^{\prime}$ increased as TTOA increased from TTOAs of 90 to $450 \mathrm{~ms}$. No other main effect or interaction reached significance [cue, $F(1,9)=2.64, p=.14$; Cue Luminance $\times$ Cue, $F(1,9)=$ $0.06, p=.81$; Cue $\times$ TTOA, $F(4,36)=0.33, p=.85$; Cue Luminance $\times$ Cue $\times$ TTOA, $F(4,36)=0.07, p=.99$ ].

Figure 11 shows the mean IOR in $d^{\prime}$ for each combination of cue luminance and SOA. Separate ANOVAs on Cue Luminance $\times$ Cue were performed at each TTOA. There were no significant effects (the largest $F$ value was 2.59).

Anticipations The anticipations for each condition are shown in Table 2. The main effect of cue luminance was significant $[F(1,9)=9.34, p<.05]$, indicating $1.85 \%$ fewer anticipations in the bright than in the dim cue trials. The main effect of TTOA was significant, $F(2.07,18.63)=$ 
Fig. 8 Mean parameters in Experiment 2: (Left) asymptote, (Middle) intercept, (Right) $1 /$ slope

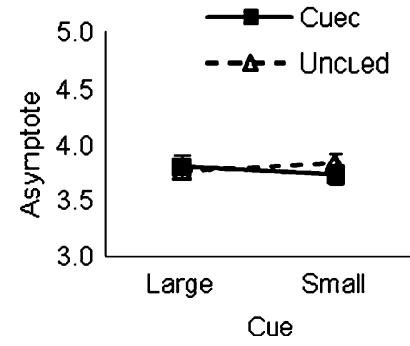

$10.10, p<.001$; anticipations increased and then stayed constant as TTOA increased. The Cue $\times$ TTOA interaction was also significant $[F(6,54)=5.75, p<.001]$.

Separate ANOVAs with the factors Cue Luminance and Cue were conducted at each TTOA. There were significant main effects of cue at TTOAs of 270 and $1,350 \mathrm{~ms}[F(1,9)=13.73, p<.01$, and $F(1,9)=7.62, p<$ $.02]$; there were, respectively, $4.73 \%$ fewer anticipations to the cued than to the uncued location at the short TTOA, and a $2.86 \%$ reversed effect at the long TTOA. There was also a reliable cue luminance effect at TTOA $900 \mathrm{~ms}[F(1$, $9)=9.79, p<.05 ; 2.95 \%$ fewer anticipations were made to the bright than to the dim cue].

Response frequencies The response frequencies are presented in Table 2. The main effect of cue luminance was significant $[F(1,9)=26.54, p<.01]$; there were $1.63 \%$ more frequent responses in the bright cue trials than in the dim cue trials. The main effect of cue was significant $[F(1,9)=$ $10.29, p<.05]$; there were $2.19 \%$ fewer responses to targets falling at cued relative to uncued locations. The main effect of TTOA was significant $[F(6,54)=9.88, p<.001]$. Hits

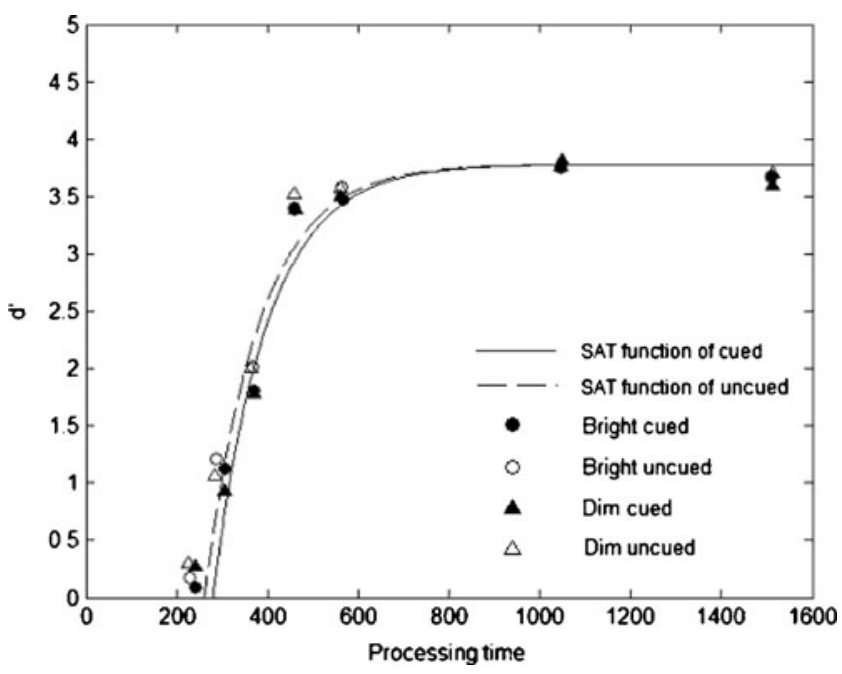

Fig. 9 Average sensitivity as a function of processing time for the critical four conditions (bright cued, bright uncued, dim cued, and dim uncued). The cued conditions have a greater intercept than the uncued conditions, which reflects delayed starting points for information processing. This effect was identical for the bright and the dim cues
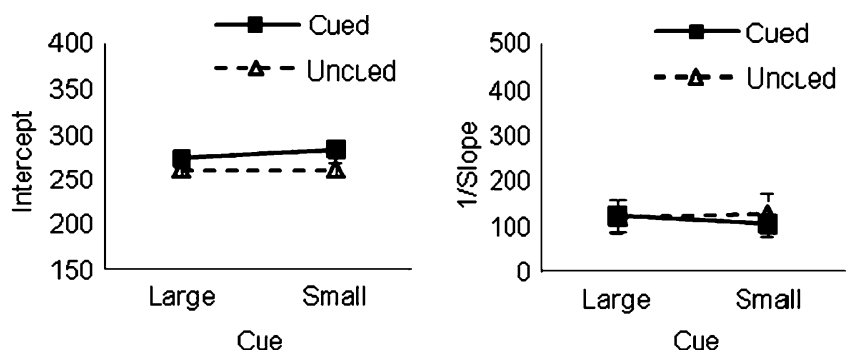

increased and then decreased as TTOA increased. The Cue $\times$ TTOA interaction was also significant $[F(6,54)=7.56$, $p<.001]$.

Separate ANOVAs with Cue Luminance and Cue as factors at each TTOA showed a significant cue main effect at TTOA $90 \mathrm{~ms}[F(1,9)=17.07, p<.01]$; there were $13.30 \%$ fewer responses to targets at the cued location than to targets at the uncued location. There was also a main effect of cue luminance at TTOA $1,350 \mathrm{~ms}[F(1,9)=7.93$, $p<.05]$; there were $6.25 \%$ more responses after bright than after dim cues.

Misses The percentages of misses are also provided in Table 2. The main effect of cue luminance was significant $[F(1,9)=26.54, p<.01]$; there were $0.02 \%$ more frequent misses to targets after a bright than after a dim cue. The main effect of cue was also significant $[F(1,9)=8.08, p<$ $.05]$ : Misses were $2.26 \%$ more frequent to targets at cued than at uncued locations. The main effect of TTOA was significant $[F(6,54)=19.43, p<.001]$. Misses first decreased and then increased as TTOA increased. The Cue $\times$ TTOA interaction was also significant $[F(6,54)=$ 9.47, $p<.001]$.

Separate ANOVAs with the factors Cue Luminance and Cue at each TTOA showed significant main effects of cue at TTOAs of 90 and $180 \mathrm{~ms}[F(1,9)=16.68, p<.01$, and $F(1,9)=5.44, p<.05 ; 12.32 \%$ and $3.75 \%$ more misses, respectively, to cued than to uncued locations]. There was also a significant cue luminance main effect at TTOA $900 \mathrm{~ms}[F(1,9)=13.33, p<.01]$, with $2.77 \%$ more misses made after bright than after dim cues.

Criterion (c) Finally, Table 2 shows the criterion measure for each condition. In this experiment, the cue main effect was unexpectedly significant $[F(1,9)=25.16, p<.05]$ : There was a slightly greater bias to "+" over " $\times$ " at the uncued location.

\section{Discussion}

The RT results were consistent with those from the first experiment in demonstrating IOR, especially at the first two TTOAs. 
Table 2 Mean tone RTs, $d^{\prime}$, percentages of anticipations, percentages of response frequencies, percentages of misses, and criterion for each condition in Experiment 2

\begin{tabular}{|c|c|c|c|c|c|c|c|c|c|}
\hline & \multirow[t]{2}{*}{ Conditions } & & \multicolumn{7}{|c|}{ TTOA (ms) } \\
\hline & & & 90 & 180 & 270 & 360 & 450 & 900 & 1,350 \\
\hline \multirow[t]{4}{*}{ Tone RTs (ms) for correct responses } & \multirow[t]{2}{*}{ Bright cue } & Cued & 150.3 & 126.7 & 100.6 & 98.2 & 117.3 & 146.4 & 158.8 \\
\hline & & Uncued & 138.1 & 106.9 & 98.0 & 98.9 & 113.9 & 146.9 & 159.7 \\
\hline & \multirow[t]{2}{*}{ Dim cue } & Cued & 149.9 & 125.4 & 99.4 & 101.2 & 111.1 & 148.3 & 161.5 \\
\hline & & Uncued & 134.5 & 104.2 & 95.1 & 99.6 & 109.8 & 147.1 & 162.9 \\
\hline \multirow[t]{4}{*}{$d^{\prime}$} & \multirow[t]{2}{*}{ Bright cue } & Cued & 0.09 & 1.12 & 1.80 & 3.38 & 3.48 & 3.76 & 3.68 \\
\hline & & Uncued & 0.17 & 1.21 & 2.01 & 3.40 & 3.59 & 3.77 & 3.67 \\
\hline & \multirow[t]{2}{*}{ Dim cue } & Cued & 0.27 & 0.93 & 1.78 & 3.39 & 3.50 & 3.82 & 3.59 \\
\hline & & Uncued & 0.30 & 1.07 & 2.00 & 3.52 & 3.57 & 3.76 & 3.70 \\
\hline \multirow[t]{4}{*}{ Anticipations (\%) } & \multirow[t]{2}{*}{ Bright cue } & Cued & 1.96 & 0.54 & 3.04 & 10.54 & 13.21 & 10.36 & 8.57 \\
\hline & & Uncued & 1.43 & 0.89 & 7.86 & 14.82 & 9.82 & 9.82 & 8.93 \\
\hline & \multirow[t]{2}{*}{ Dim cue } & Cued & 1.43 & 1.07 & 3.57 & 12.50 & 13.39 & 13.39 & 17.50 \\
\hline & & Uncued & 1.79 & 1.96 & 8.21 & 14.64 & 14.11 & 12.68 & 11.43 \\
\hline \multirow[t]{4}{*}{ Response frequencies (\%) } & \multirow[t]{2}{*}{ Bright cue } & Cued & 65.71 & 80.18 & 85.36 & 82.14 & 75.54 & 74.82 & 69.11 \\
\hline & & Uncued & 80.00 & 85.00 & 81.07 & 78.04 & 79.82 & 73.04 & 66.43 \\
\hline & \multirow[t]{2}{*}{ Dim cue } & Cued & 66.07 & 79.46 & 83.39 & 78.57 & 75.71 & 74.29 & 59.11 \\
\hline & & Uncued & 78.39 & 83.21 & 81.25 & 79.82 & 76.96 & 73.21 & 63.93 \\
\hline \multirow[t]{4}{*}{ Misses (\%) } & \multirow[t]{2}{*}{ Bright cue } & Cued & 29.82 & 15.89 & 9.11 & 6.43 & 10.89 & 14.82 & 21.07 \\
\hline & & Uncued & 16.61 & 11.07 & 10.18 & 6.43 & 10.18 & 16.79 & 23.93 \\
\hline & \multirow[t]{2}{*}{ Dim cue } & Cued & 28.93 & 15.54 & 11.61 & 7.86 & 10.00 & 11.96 & 25.00 \\
\hline & & Uncued & 17.50 & 12.86 & 9.11 & 4.82 & 8.75 & 14.11 & 25.00 \\
\hline \multirow[t]{4}{*}{ Criterion } & \multirow[t]{2}{*}{ Bright cue } & Cued & .01 & .06 & .01 & -.15 & -.04 & -.03 & .04 \\
\hline & & Uncued & .14 & -.02 & .05 & -.05 & .01 & -.04 & .00 \\
\hline & \multirow[t]{2}{*}{ Dim cue } & Cued & .15 & .04 & .02 & -.06 & -.04 & .02 & .03 \\
\hline & & Uncued & .09 & .01 & .11 & .05 & -.02 & .07 & -.04 \\
\hline
\end{tabular}

The columns in bold indicate that ANOVAs for the respective TTOAs found significant effects (see the text for details). Note that the criterion value in a discrimination task only indicates a bias towards a particular response. Here, positive criterion values indicate a response bias towards "+," and negative values indicate a bias towards " $\times$ ".

Unlike the first experiment, the present experiment yielded two distinct SAT functions for cued and uncued trials. IOR delayed the point in time when the target information started to accumulate above chance (the intercept parameter). This is consistent with Ivanoff and Klein's (2006) Experiment 2 (Fig. 5 in their article). However, in contrast to our results, their pattern, due in part to the limited number of TTOAs, could be attributed to either an intercept effect or an effect on the asymptote of the sensitivity function. Our data, however, provide a clear indication for an effect of IOR on the dynamics of perceptual coding, indexed by the intercept parameter on the SAT function.

In addition, the data from this experiment again point to a more conservative criterion being adopted for targets at the cued than at the uncued location. This was reflected by fewer hits and more misses to targets falling on cued rather than uncued locations.
Fig. 10 IOR on RTs in Experiment 2. Positive values indicate IOR

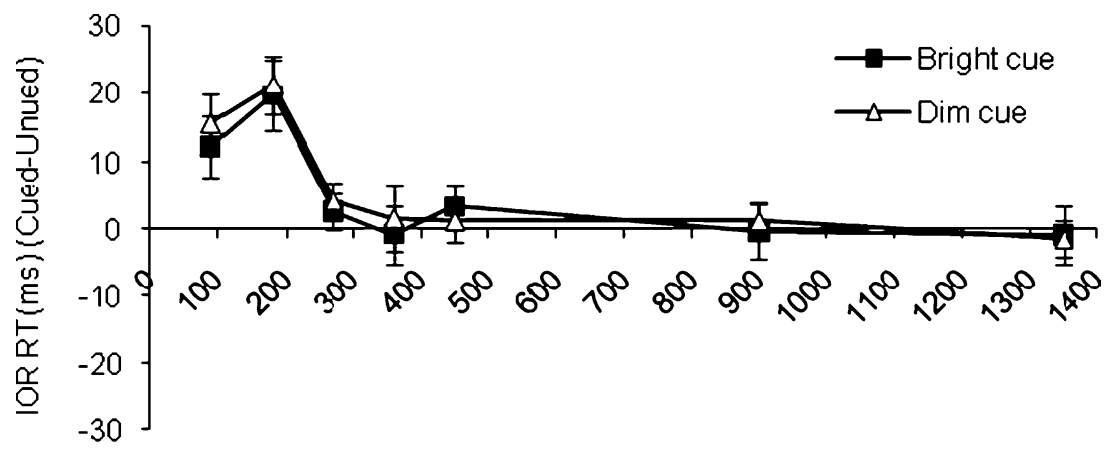

Processing time (ms) 
Fig. 11 IOR on $d^{\prime}$ in Experiment 2. Positive values indicated lower $d^{\prime}$ at the cued location

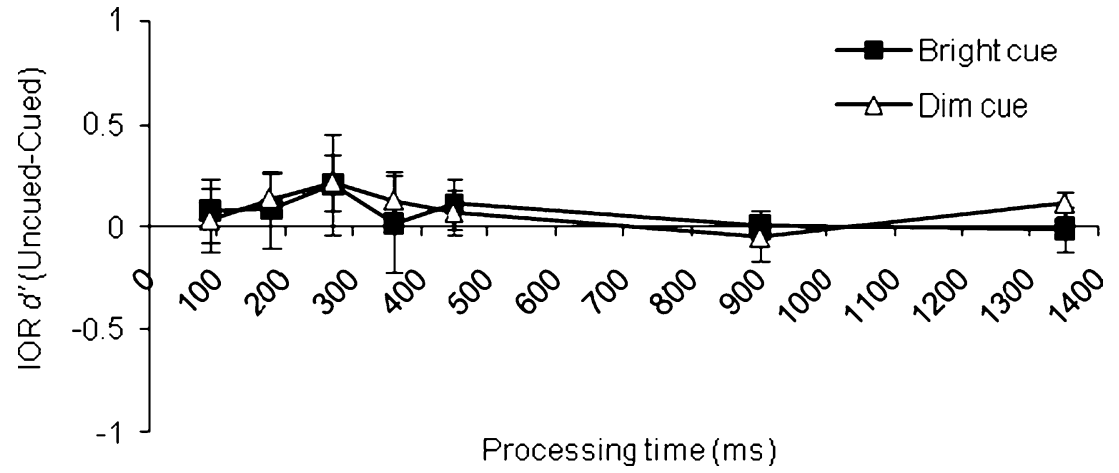

Given the apparent differences in the SAT functions for Experiment 2 as compared with the earlier experiment, a formal comparison was undertaken.

\section{Comparison across experiments}

\section{SAT function}

The parameters were analysed across two experiments with Experiment as the between-subjects factor. The ANOVA revealed a Cue $\times$ Experiment interaction for the intercept parameter $[F(1,25)=6.71, p<.05]$. This interaction was driven by the main effect of the cue being significant only in Experiment 2 (a greater intercept for cued than for uncued trials). Similar analyses on the other SAT parameters revealed no significant effects (all $p \mathrm{~s}>.1$ ).

\section{Tone RT}

A mixed ANOVA was conducted to compare tone RTs across experiments. There was a significant main effect of cue $[F(1,25)=34.82, p<.001$; RTs for cued $>$ uncued locations, 5.21-ms effect], as well as a reliable TTOA effect $[F(3.77,94.21)=52.31, p<.001]$. There was also a Cue $\times$ TTOA interaction $[F(6,150)=15.73, p<.001]$ driven by the decreased IOR effect as the TTOA lengthened.

\section{Sensitivity $\left(d^{\prime}\right)$}

A mixed ANOVA revealed a significant TTOA effect $[F(4$, $100)=267.10, p<.001]$ and a significant Cue $\times$ Experiment interaction $[F(1,25)=9.12, p<.01]$ (see Fig. 12). In Experiment 1, sensitivity increased at the cued relative to the uncued location, while the data went (nonsignificantly) in the opposite direction in Experiment 2.

\section{Anticipations}

Only those effects involving cue or experiment are listed here (the same holds for the response frequency and miss data). The main effect of cue was significant $[F(1,25)=$ $7.23, p<.05]$, as was the Cue $\times$ TTOA interaction $[F(3.95$, $98.81)=6.91, p<.001]$. There were fewer anticipations made to targets at the cued than at the uncued location, especially at TTOAs of 180, 270, and $360 \mathrm{~ms}$. The Cue Luminance $\times$ Experiment interaction was also significant $[F(1,25)=4.95, p<.05]$, driven by the effect of cue luminance being significant only in Experiment 2 (see the separate analysis for this experiment). There were no other significant effects involving cue or experiment.

\section{Response frequency}

The main effect of the cue was significant $[F(1,25)=$ $14.81, p<.001]$, along with the Cue $\times$ TTOA interaction $[F(6,150)=12.10, p<.001]$. The response frequency was lower to targets at the cued relative to the uncued location (especially at TTOAs of 90 and $180 \mathrm{~ms}$; however, there was a reverse effect at TTOA $270 \mathrm{~ms}$ ). There were no any significant effects involving either the cue or the experiment.

\section{Misses}

Both the main effect of the cue $[F(1,25)=21.85, p<.001]$ and the Cue $\times$ TTOA interaction $[F(6,150)=13.05, p<$ $.001]$ were significant. There were more misses to targets at cued than at uncued locations (especially at TTOAs of 90 and $180 \mathrm{~ms})$. In addition, the TTOA $\times$ Experiment

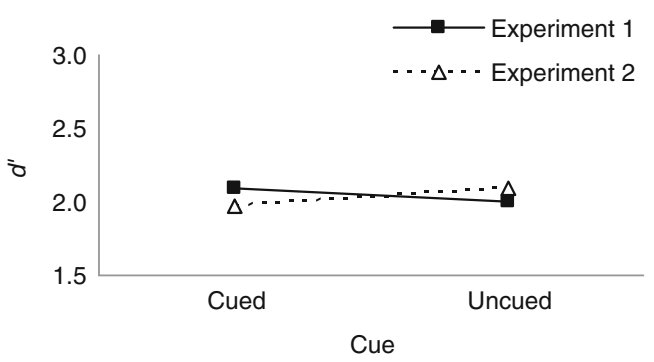

Fig. 12 Average $d^{\prime}$ to the cued and the uncued targets in each experiment. In Experiment 1, a cue increased the sensitivity at the cued location. However, in Experiment 2, there was no any significant difference between the cued and the uncued locations 
interaction was significant $[F(6,150)=3.07, p<.01]$. There were no significant effects involving cue or experiment.

Summary of the comparisons

Table 3 summarises the results of Experiments 1 and 2. The comparison of the SAT parameters confirmed that Experiments 1 and 2 differed in terms of their SAT functions for cued and uncued trials. In Experiment 1, for which a brief target exposure was used, there was no reliable effect of the cue on the parameters of the SAT function, and all the other evidence pointed to an effect of IOR on the response criterion adopted (while perceptual sensitivity tended to be higher at cued locations, RTs were slower, the rate of anticipatory responses was lower, and misses were greater). In contrast, Experiment 2 showed an effect of the cue on the intercept of the SAT function (while sensitivity did not show any significant change, RTs were slower), suggesting that IOR delayed perceptual processing in this case. In addition, there was evidence of conservative responses (the response frequency was lower and misses were greater at cued than at uncued locations), as was also found for Experiment 1. The data indicate that, with short target exposures, IOR affects the response criterion adopted. With longer exposure, some effects on perceptual processing emerge, in addition to effects on the response criterion.

\section{General discussion}

This article reports two experiments that explored the IOR effect using the SAT methodology, which allows the experimenter to jointly control RTs and accuracy when participants respond to targets. The specific implementation of the SAT methodology employed here follows the experimental procedure devised by Ivanoff and Klein (2006), which entails setting RTs via a response window.

Using the SAT methodology, the study explored how IOR is implemented within the framework of SDT. According to SDT, IOR may operate at the stage of perceptual coding, or decision, or both. Effects of IOR on perceptual coding are indicated by a decrease of sensitivity for targets falling at cued relative to uncued locations. In contrast evidence for increased sensitivity at the cued location is consistent with a criterion shift account. As noted in the introduction, there is empirical support for both proposals in the literature. We compared effects with limited and unlimited target presentation times and showed that different effects emerged in these conditions (Table 3). With short exposures, there was good evidence for IOR reflecting a change in the response criterion. On the other hand, Experiment 2 provided evidence for a change in the SAT function consistent with delayed perceptual processing at the cued location, in addition to a conservative criterion shift.

We also found that, across the experiments, there was a small but significant IOR effect for the short TTOAs. It is possible that, when fast responses were required, participants might have used IOR to perform the task more efficiently, whereas when they had more time to prepare a response, IOR offered less help for improving performance, especially when perceptual information had reached an asymptote. Experiment 2 essentially replicated Ivanoff and Klein's (2006) findings, while at the same time enabling us to fit an SAT function to the results. Finally, note that all of our results were unaffected by different levels of cue and target luminance, indicating a degree of generality with respect to these factors.

Taken together, our experimental results suggest a hybrid account in which there is a criterion shift, present with both short and prolonged target exposures, plus an effect on attention/perception when target presentation is prolonged. Past research had suggested that IOR is realised in a combination of attentional and motor components. Abrams and Dobkin (1994) compared the time to make an eye movement instructed by either peripheral or central targets after a peripheral cue. IOR was found to be larger in the peripheral than in the central condition. ${ }^{5}$ They argued that, with a central cue, programming an eye movement evokes the motor component of IOR. In contrast, a peripheral cue enhances perception as well as cueing the eye movement, so that both perceptual/attentional and motor components are present-which then leads to the increased IOR effect. Furthermore, Kingstone and Pratt (1999) investigated IOR in both stimulus localisation and identification tasks, and their results also supported both attentional and motor accounts of IOR. Evidence for an attentional component came from the finding that IOR was obtained for both localisation and identification, as Kingstone and Pratt argued that motor effects should be evident on stimulus localisation and not identification. These authors also obtained evidence for a contribution of an oculomotor response to IOR, with IOR increasing when eye movements were executed as compared to when eye movements were withheld. Similarly, there might have been some contributions from microsaccades (for more details, see Betta, Galfano, \& Turatto, 2007; Galfano, Betta, \& Turatto, 2004).

\footnotetext{
${ }^{5}$ Challenging this finding, Taylor and Klein (2000) found equivalent IOR when they explored these same two conditions. The discrepancy was recently resolved by Hilchey, Klein, and Ivanoff (2011) who noted that by presenting peripheral and central arrow targets in different blocks of trials, Abrams and Dobkin (1994) gave their participants an opportunity to focus their attention so exclusively on fixation in the arrow condition that the peripheral cues might be filtered out, thereby reducing the IOR they generate. When Hilchey et al. (2011) replicated the Abrams \& Dobkin methods while randomly intermixing the central and peripheral targets (as did Taylor \& Klein, 2000) they found equivalent IOR with the two target types.
} 
Table 3 Summary of the results for Experiments 1 and 2

\begin{tabular}{llcc}
\hline & Experiment 1: Brief Target & Experiment 2: Unlimited Target & Cross Experiments \\
\hline Parameters of SAT function & $\begin{array}{c}\text { Not significant on any } \\
\text { parameters }\end{array}$ & $\begin{array}{c}\text { Intercept parameter: } \\
\text { cued }>\text { uncued* }\end{array}$ & $\begin{array}{c}\text { intercept parameter: cue } \times \\
\text { experiment* }\end{array}$ \\
RT and $d^{\prime}$ & $\begin{array}{c}\text { RT: cued }>\text { uncued**; } \\
d^{\prime}: \text { cued }>\text { uncued* }\end{array}$ & $\begin{array}{c}\text { RT: cued }>\text { uncued*; } \\
d^{\prime}: \text { not significant }\end{array}$ & $\begin{array}{c}\text { RT: cued }>\text { uncued**; } \\
d^{\prime}: \text { Cue } \times \text { Experiment** }\end{array}$ \\
$\begin{array}{l}\text { Response categories (anticipation; } \\
\text { response frequency; miss) }\end{array}$ & $\begin{array}{c}\text { Anticipations: cued }<\text { uncued*; } \\
\text { misses: cued }>\text { uncued }\end{array}$ & $\begin{array}{c}\text { Response frequencies: } \\
\text { cued }<\text { uncued*; } \\
\text { misses: cued }>\text { uncued* }\end{array}$ & $\begin{array}{c}\text { Anticipations: cued }<\text { uncued*; } \\
\text { Responses frequency: } \\
\text { cued }<\text { uncued**; } \\
\text { misses: cued }>\text { uncued** }\end{array}$
\end{tabular}

${ }^{*} p<.05 .{ }^{* *} p<.01$.

The question remains, however, of why both components of IOR may have operated here when the target presentation time was unlimited, while, with a limited presentation time, participants relied only on a criterion shift. Here, we can only speculate. When the target is presented indefinitely, there is opportunity for accrual sensitivity to reach its peak, even with a delayed starting point (after the target presentation). In contrast, when the target presentation time is brief, any delay may result in an unrecoverable loss of target information. As a consequence, when participants know that the stimulus will be presented briefly, they may be able to compensate for an initial intercept disadvantage due to IOR. For example, compensation may come in the form of earlier sampling of perceptual evidence, relative to when the target information is available for much longer, and this overcomes the effect of the perceptual component of IOR. Therefore, the attentional/perceptual component to IOR is only revealed when the target presentation time is long. Further research could investigate IOR when the target presentation times are unpredictable or when participants have biased expectations as to the target presentation, to assess whether a strategic change in perceptual processing is possible.

This explanation of our results is based on the assumption that the perceptual impairment operates in addition to the motor effect. In contrast, work by Taylor and Klein (2000), Hunt and Kingstone (2003), and recently by Chica, Taylor, Lupiáñez, and Klein (2010) has suggested that two different "flavours" of IOR are generated under different circumstances (i.e., two components of IOR are isolated): An attention/perceptual IOR (impaired processing at the peripheral locations) is generated when the oculomotor system is tonically inhibited, while a motor IOR (motoric bias in responding) is generated when the oculomotor system is activated. This hypothesis is based on a series of experiments by Taylor and Klein, who used a combination of peripheral and central cues and targets.
Participants were required to either ignore or make manual or saccadic responses to the cues. To the targets, participants had to make either manual or saccadic responses. Taylor and Klein found that, when no eye movements were made to either the cue or the target, IOR only occurred for manual responses to peripheral targets, and not to central targets. Under the latter condition, there was no motoric component linked to an eye movement and no attentional/ perceptual component, as the target appeared at fixation rather than at the location signalled by the cue. However, even without eye movements (and no motor component), there was IOR to peripheral targets, consistent with attentional/perceptual IOR when the oculomotor system was tonically inhibited, while the motor component contributed when eye movements were made. Importantly, when eye movements were made, the IOR effect to central targets (the motor component alone) was at least as large as that to peripheral targets (the attentional/perceptual and motor components), offering no support to the additivecomponents view. The idea that two "flavours" of IOR develop according to whether an eye movement occurs might be used to account for why the mechanism underlying the IOR effect (motor/criterion versus perception/attention) might vary with target duration. ${ }^{6}$ Although

\footnotetext{
${ }^{6}$ When the targets were briefly presented, participants might not even have noticed the incorrect eye movements, and, thus, the oculomotor system would not be strongly inhibited. Therefore, a pure motor/ criterion effect was observed. However, with the prolonged target presentation, when eye movements were made, participants were able to see the target and became aware of their failure to follow the instructions. This might have initiated a period during which the oculomotor system would have been inhibited, resulting in perceptual/ attentional IOR. However, eventually, participants stopped inhibiting eye movements - for instance, due to fatigue - and subsequently reinstated the motor IOR. Therefore, it is possible that the evidence for both perceptual processing delay and criterion shift results from a mixture of states across trials.
} 
participants were instructed not to make eye movements, eye movements were not monitored and might have been made unknowingly. These speculations have to be explored in further experiments by using eyetracking.

In any case, our results indicate that target exposure duration is an important factor determining the locus at which IOR affects processing. Interestingly, unlike Handy et al. (1999), who showed that accuracy decreases at longer RTs, we found that target information continued to improve even after the target disappeared (with short exposures, in Exp. 1). Although this appears to be inconsistent with the assumption that, with short target exposures, information about the target decays (e.g., Ivanoff \& Klein, 2006), it must be kept in mind that we did not mask the targets after their brief presentation. Moreover, the asymptote and rate parameters of the SAT function did not differ across the two experiments, suggesting that the briefly presented target provided the same perceptual information as targets given prolonged exposure. Hence, it is possible that $80 \mathrm{~ms}$ was long enough to initiate early visual processing sufficiently to ensure that information accrual continued beyond the presentation time. We note here that Handy et al. (1999) presented a mask after exposing the target, and this would have disrupted information acquisition. A second apparently discrepant finding here, relative to the literature, is that studies with brief target presentation times (Cheal et al., 1998; Handy et al., 1999; Lupiáñez et al., 1997) have found support for attentional/perceptual accounts of IORdirectly contradicting our findings. However, as we pointed out, the accuracy measured in these studies might have been affected by the decay of target information, which was not apparent here.

In sum, the data presented here point to there being different factors involved in IOR: It reflects a criterion shift with both brief and longer stimulus exposures, whereas, with longer exposures, there are also effects on attention/ perception. The SAT procedure provides a powerful tool for pulling these different effects apart.

Acknowledgment The authors thank the Experimental Psychology Society (EPS) for funding Y.Z.'s 5-week visit to R. M. Klein's laboratory. Some of the research presented was made possible by NSERC Discovery Grants to R.K. and to J.I.

\section{References}

Abrams, R. A., \& Dobkin, R. S. (1994). Inhibition of return: Effects of attentional cuing on eye movement latencies. Journal of Experimental Psychology. Human Perception and Performance, 20, 467-477. doi:10.1037/0096-1523.20.3.467

Betta, E., Galfano, G., \& Turatto, M. (2007). Microsaccadic response during inhibition of return in a target-target paradigm. Vision Research, 47, 428-436.
Brown, S. D., \& Heathcote, A. (2008). The simplest complete model of choice response time: Linear ballistic accumulation. Cognitive Psychology, 57, 153-178. doi:10.1016/j.cogpsych.2007.12.002

Carrasco, M., \& McElree, B. (2001). Covert attention accelerates the rate of visual information processing. Proceedings of the National Academy of Sciences, 98, 5363-5367. doi:10.1073/ pnas. 081074098

Cheal, M., Chastain, G., \& Lyon, D. R. (1998). Inhibition of return in visual identification tasks. Visual Cognition, 5, 365-388. doi:10.1080/713756787

Chica, A. B., Taylor, T. L., Lupiáñez, J., \& Klein, R. M. (2010). Two mechanisms underlying inhibition of return. Experimental Brain Research, 201, 25-35.

Dosher, B. A., Han, S. M., \& Lu, Z. L. (2004). Parallel processing in visual search asymmetry. Journal of Experimental Psychology. Human Perception and Performance, 30, 3-27.

Farrell, S., Ludwig, C. J. H., Ellis, L. A., \& Gilchrist, I. D. (2010). Influence of environmental statistics on inhibition of saccadic return. Proceedings of the National Academy of Sciences, 107, 929-934. doi:10.1073/pnas.0906845107

Folk, C. L., Remington, R. W., \& Johnston, J. C. (1992). Involuntary covert orienting is contingent on attentional control settings. Journal of Experimental Psychology. Human Perception and Performance, 18, 1030-1044. doi:10.1037/0096-1523.18.4.1030

Galfano, G., Betta, E., \& Turatto, M. (2004). Inhibition of return in microsaccades. Experimental Brain Research, 159, 400-404.

Giordano, A. M., McElree, B., \& Carrasco, M. (2009). On the automaticity and flexibility of covert attention: A speed-accuracy trade-off analysis. Journal of Vision, 9(3), 30:1-10. doi: 10.1167/ 9.3.30

Green, D. M., \& Swets, J. A. (1966). Signal detection theory and psychophysics. New York: Wiley.

Handy, T. C., Jha, A. P., \& Mangun, G. R. (1999). Promoting novelty in vision: Inhibition of return modulates perceptual-level processing. Psychological Science, 10, 157-161. doi:10.1111/14679280.00124

Hilchey, M. D., Klein, R. M. \& Ivanoff, J. (2011, May) Perceptual and motor IOR: Components or flavours? Presented at the Annual Meeting of the Vision Sciences Society (Naples, Florida).

Hunt, A. R., \& Kingstone, A. (2003). Inhibition of return: Dissociating attentional and oculomotor components. Journal of Experimental Psychology. Human Perception and Performance, 29, 1068-1074. doi:10.1037/0096-1523.29.5.1068

Ivanoff, J., \& Klein, R. M. (2001). The presence of a nonresponding effector increases inhibition of return. Psychonomic Bulletin \& Review, 8, 307-314. doi:10.3758/BF03196166

Ivanoff, J., \& Klein, R. M. (2003). Orienting of attention without awareness is affected by measurement-induced attentional control settings. Journal of Vision, 3(1), 4:32-40. doi: 10.1167/3.1.4

Ivanoff, J., \& Klein, R. M. (2004). Stimulus-response probability and inhibition of return. Psychonomic Bulletin \& Review, 11, 542550. doi:10.3758/BF03196608

Ivanoff, J., \& Klein, R. M. (2006). Inhibition of return: Sensitivity and criterion as a function of response time. Journal of Experimental Psychology. Human Perception and Performance, 32, 908-919. doi:10.1037/0096-1523.32.4.908

Kingstone, A., \& Pratt, J. (1999). Inhibition of return is composed of attentional and oculomotor processes. Perception \& Psychophysics, 61, 1046-1054.

Klein, R. M., \& Taylor, T. L. (1994). Categories of cognitive inhibition with reference to attention. In D. Dagenbach \& T. H. Carr (Eds.), Inhibitory mechanisms in attention memory and language (pp. 113-150). San Diego: Academic Press.

Liu, C. C., \& Smith, P. L. (2009). Comparing time-accuracy curves: Beyond goodness-of-fit measures. Psychonomic Bulletin \& Review, 16, 190-203. 
Lupiáñez, J., Milan, E. G., Tornay, F. J., Madrid, E., \& Tudela, P. (1997). Does IOR occur in discrimination tasks? Yes, it does, but later. Perception \& Psychophysics, 59, 1241-1254. doi:10.3758/ BF03214211

Macmillan, N. A., \& Creelman, C. D. (2005). Detection theory: A user's guide (2nd ed.). Mahwah, NJ: Erlbaum.

McCormick, P. A., \& Francis, L. (2005). Speed-accuracy tradeoff operator characteristics of endogenous and exogenous covert orienting of attention. The Scientific World, 5, 128-146. doi:10.1100/tsw.2005.20

Miller, J., Sproesser, G., \& Ulrich, R. (2008). Constant versus variable response signal delays in speed-accuracy tradeoffs: Effects of advance preparation for processing time. Perception \& Psychophysics, 70, 878-886. doi:10.3758/PP.70. 5.878

Posner, M. I. (1980). Orienting of attention. Quarterly Journal of Experimental Psychology, 32, 3-25. doi:10.1080/003355 58008248231

Posner, M. I., \& Cohen, Y. (1984). Components of visual orienting. In H. Bouma \& D. G. Bouwhuis (Eds.), Attention and performance $X$ : Control of language processes (pp. 531-556). Hove, U.K.: Erlbaum.

Posner, M. I., Rafal, R. D., Choate, L. S., \& Vaughan, J. (1985). Inhibition of return: Neural basis and function. Cognitive Neuropsychology, 2, 211-228. doi:10.1080/026432985082 52866

Prime, D. J., \& Ward, L. M. (2004). Inhibition of return from stimulus to response. Psychological Science, 15, 272-276. doi:10.1111/ j.0956-7976.2004.00665.x
Reed, A. V. (1973). Speed-accuracy trade-off in recognition memory. Science, 181, 574-576. doi:10.1126/science.181.4099.574

Reuter-Lorenz, P. A., Jha, A. P., \& Rosenquist, J. N. (1996). What is inhibited in inhibition of return? Journal of Experimental Psychology. Human Perception and Performance, 22, 367-378. doi:10.1037/0096-1523.22.2.367

Snodgrass, J. G., \& Corwin, J. (1988). Pragmatics of measuring recognition memory: Applications to dementia and amnesia. Journal of Experimental Psychology. General, 117, 34-50. doi:10.1037/0096-3445.117.1.34

Swets, J. A., Green, D. M., Getty, D. J., \& Swets, J. B. (1978). Signal detection and identification at successive stages of observation. Perception \& Psychophysics, 23, 275-289. doi:10.3758/ BF03199711

Taylor, T. L., \& Klein, R. M. (2000). Visual and motor effects in inhibition of return. Journal of Experimental Psychology. Human Perception and Performance, 26, 1639-1656. doi:10.1037/00961523.26.5.1639

Wickelgren, W. A. (1977). Speed-accuracy tradeoff and information processing dynamics. Acta Psychologica, 41, 67-85. doi:10.1016/0001-6918(77)90012-9

Yantis, S., \& Hillstrom, A. P. (1994). Stimulus-driven attentional capture: Evidence from equiluminant visual objects. Journal of Experimental Psychology. Human Perception and Performance, 20, 95-107. doi:10.1037/0096-1523.20.1.95

Yantis, S., \& Jonides, J. (1984). Abrupt visual onsets and selective attention: Evidence from visual search. Journal of Experimental Psychology. Human Perception and Performance, 10, 601-621. doi:10.1037/0096-1523.10.5.601 